\title{
Discussing of Deformation of Additive Manufacturing due to External Bending
}

\author{
Ya-Ju Lin ${ }^{1, a}$, Jia-Chang Wang ${ }^{2, b}$, Cherng-Yuh Su ${ }^{3, c}$, Che-Hua Yang ${ }^{4, d}$ \\ ${ }^{1,2,3}$ Department of Mechanical Engineering, National Taipei University of Technology, Taipei, \\ TAIWAN \\ ${ }^{4}$ College of Mechanical and Electrical Engineering, National Taipei University of Technology, \\ Taipei, TAIWAN

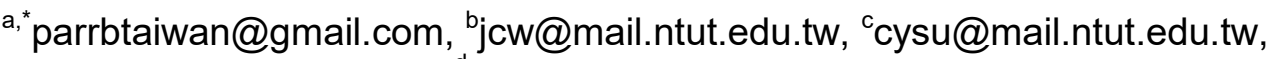 \\ 'chyang@ntut.edu.tw
}

Keywords: Additive-Manufacturing, 3D Printing, Ceramic, Multilayer, Residual Stress

\begin{abstract}
Default and deformations are some of issues for Additive manufacturing, as known as the 3D printing. When it processes multilayer thin films stacking, this process will introduce higher residual stress that causes deformation. This phenomenon makes the product faulty such as delamination, crack and blisters. The reason could be included ununiformed casting, temperature, cubical contraction etc. However, it is not easily to seem the difference form the films in the present. For ceramic materials, if deformation was existed at interface between layers, the crack would appear after the sintering was completed. For elastic material, the film will be bended, if the structure of product is unstable or the thickness is not thick enough to resist bending. In order to observe the deformation from material, residual stress and external bending must be considered. The purpose of the present study is to find the best approximation of formula for $3 \mathrm{D}$ printing and to predict the residual stress for the products. Later, the producing process could be adjusted until the most deformation is eliminated. The methods for present study are considered for two multilayer formulas. One is derived from Hsueh's closed-form, the anther is Timoshenko Theory. The results are combined simulation and experimental for two material and their accuracy is examined. Except considering the effect of the thermal stress, the acceptability of assuming for analyses in the 3D printing system is discussed.
\end{abstract}

\section{Introduction}

Additive manufacturing (AM), an exclusive mechanical technology, has introduced in past few decade. It is different from conventional manufacturing process that limited for creating a small and complex product. There are fewer restrictions for use of machining, molding, costing. Its potential can benefit designer to improve their artwork easily. The model was designed form Computer Aided Design (CAD) as 3D digital data and completed by AM. It simplified many complex preprocess steps from conventional manufacturing such as preprocess and post process. Therefore, the material waste and production time can be reduced. Based on different of material was used for AM, the produce of process can be widely different. For metal additive manufacturing, the processes contain power bed process, blown powder and wire feed processes [1].

Ceramic material is hardly to fabricate a small and complex product when using conventional manufacturing process. AM is an alternative way to product a high quality product such as creating dentures. Also, it does not need to prepare molds for model as preprocess. Therefore, to improve those advantage such as strengths and efficiency, there are many kinds of 3D-Printing devices were introduced recently such as Sterelolithography(SL) (Griffith et al., 1996; Hinczewski et al., 1998; Chartier et al., 2002) ; Michosterelothograph (In-Baek Park et al., 2009; Young-Myoung Ha et al., 2010); Selective Laser Sintering (SLS) (Subramanian et al., 1995; Liu et al., 2007); Fused Deposition of Ceramics (FDC) (Agarwala et al., 1996); Laminated Object Manufacturing (LOM) (Klosterman et al., 1998), Slurry-Based Three Dimensional Printing (S3DP) (Grau et al., 1997). 
However, there are unexpected and irregular deformation appeared when fabricate the product layer by layer. The reason can be caused by incomplete adhesion between layers or uncompleted rigidified surface. Fig. 1 shows deformation in the alumina ceramic product. Bending due to shrinkage is also happened to both materials. Fig. 2 illustrated the bending stress generate in each layer due to shrinkage and illustrated layer additive process section where (a) casting slurry on the platform, (b) digital light processing (DLP) projector exposed onto object. The exposed area is consolidated and constrained form lower layer, (c) compressive stresses are then imposed on the individual layers to achieve displacement compatibility, (d) the asymmetric stresses system occurred bending product. Therefore, the process of AM can be described as a multilayer system. The multilayered systems are strongly influenced by residual stress. Most analyses are generally based on classical beam bending theory and strain continuity at interface between layers is required [2-3]. Hsueh presented an analysis for multilayer systems that the deformation was due to external bending [4].

Solvent-based slurry stereolithogtaphy (3S) [5] was the main AM for all experimental specimens in this paper. The purpose of the present study is to observe shrinkage of $3 \mathrm{~S}$ product in multilayer systems. In order to detect inner stress and measure deformation, it would be necessary to observe eigenstrain. A semi-empirical procedure for observing eigenstrain from additive manufacturing was presented. Based on detected curvature form bending system, the eigenstrain can be simply defined. The predicted and measured result can be compared. The material was used photopolymer resin and alumina ceramic. Both materials were mixed photoinitiator that changes properties when exposed to the ultraviolet light. All products were made at room temperature. For alumina ceramic, sintering process was completed after printing process.

\section{Method}

The interfacial shearing stress can be predicts form Thimoshenko's joined beams bimetal thermostat model [6]. Sherman et al. extend theory to multilayer system as the eigenstrain-thickness function [7]. understand the bending stress in multilayer material for 3D printing, the theoretical analysis was considered. The assumption of eigenstrain-thickness function for multilayer film addressed as follows:

1. The material for each layer is homogeneous, isotropic and linearly elastic.

2. The thickness of each layer is uniform.

3. Perfectly bonded interface exists between each layer.

4. The peeling stress assumed as zero.

For all $\mathrm{n}^{\text {th }}$ of linear elastic multilayer films, each denoted by subscript $i$, which has their own thermal eigenstrain, $\varepsilon_{i}^{\text {thermal }}$, eigenstrain due to growth within the layers $\varepsilon_{i}^{\text {bend }}$, and the axial eigenstrain $\varepsilon_{i}^{\text {axial }}$. Thus the total eigenstrain, $\varepsilon_{i}^{\text {total }}$, as shown as below:

$$
\varepsilon_{i}^{\text {total }}=\varepsilon_{i}^{\text {thermal }}+\varepsilon_{i}^{\text {bend }}+\varepsilon_{i}^{a x i a l}
$$

For each of thickness force and moment is defined as $P, M$. The resultant force is zero due to the uniform strain component. Moreover, the sum of bending moment is equilibrium. Therefore the equilibrium equation descripted as below:

$$
\begin{aligned}
& \sum_{n=1}^{n} P_{i}=0 \\
& \sum_{i=1}^{n} M_{i}-\sum_{i=1}^{n-1} P_{i}\left[\frac{t_{i}-t_{n}}{2}+\sum_{k=i+1}^{n-1} t_{k}\right]=0
\end{aligned}
$$


where $t$ is layer thickness. The moment for each layer is related to the curvature and contained bending rigidity as shown as below:

$$
\sum_{i=1}^{n} M_{i}=\sum_{i=1}^{n-1} M_{i}+M_{n}=\frac{1}{\rho\left(1-v_{i}^{2}\right)}\left(\sum_{i=1}^{n-1} E_{i} I_{i}+E_{n} I_{n}\right)
$$

where $\rho, v, E, I$ is radius of curvature, Poisson's ratio, Young's modulus and Moments of inertia respectively.

The unit elongation between two layers which along the $n-1$ interfaces

$$
\varepsilon_{i-1}^{\text {tota }}+\frac{P_{i-1}}{E_{\bar{i}-1} t_{i-1}}+\frac{t_{\bar{i}-1}}{2 \rho}=\varepsilon_{i}^{\text {total }}+\frac{P_{\bar{i}}}{E_{i} t_{\bar{i}}}-\frac{t_{\bar{i}}}{2 \rho}
$$

combined Eq.3, 4, 5, then the curvature can be defined as

$$
\frac{1}{\rho}=\frac{\Delta \varepsilon^{\text {total }}}{P_{i-1}-\frac{P_{i}}{E_{i-1} t_{i-1}}+t_{i}+t_{i-1}}
$$

The discrete values of residual stress before unconstrained is calculated from

$$
\sigma_{i}=\frac{P_{i}}{t_{i}}+\frac{t_{i} E}{2 \rho}
$$

\section{Experimental measurement}

There are two measurements to measure Young's modulus for materials. For specimen of photopolymer resin, Young's modulus can be measured by using tensile test. The other material couldn't be detected correctly form tensile test. For specimen of alumina ceramic, the ultrasound signal generator is an alternative method to measure Young's modulus, as shown in Fig. 4. The velocity of a transverse wave $\left(\mathrm{C}_{\mathrm{T}}\right)$ and longitudinal wave $\left(\mathrm{C}_{\mathrm{L}}\right)$ can be obtained by using ultrasound signal generator. The equation of transverse wave and longitudinal wave can be founded below [8].

$$
C_{T}=\sqrt{\frac{E}{2 D(1+v)}}, C_{L}=\sqrt{\frac{\lambda+2 G}{D}}
$$

where $\mathrm{D}$ is density of the specimen. $\mathrm{G}$ and $\lambda$ is shear modulus, wavelength respectively. Therefor Young's modulus can be calculated from this equation shown as:

$$
G=\frac{E}{2(1+v)}
$$

According to bending system, the total curvature needs to obtain by experimental data. The 3D measurement system is a way to measure the total curvature of specimens for both materials.

\section{Result}

Table 1 lists 6 different total thickness of photopolymer resin. The specimen of photopolymer resin is designed as $30 \mathrm{~mm}$ (wide) x $50 \mathrm{~mm}$ (length). The thickness is $50 \mu \mathrm{m}$ for each layer. After completed printing process, specimens exposed in the UV light again. This post-process is to make sure the boundary and surface are fully solidified binder and the specimens were not dissolved in methanol at 
room temperature. After irradiating process, the shrinkage of specimens is around in $90 \%$ to $95 \%$. The Young's modulus is $294 \mathrm{MPa}$, which was obtained by using tensile test. The measurements of curvature, which presented in black color in Fig 5., shows declined when total thickness of specimens was increased. Using curvature data, the eigenstrain can be obtained individually form eigenstrain-thickness function. The result shows in Fig. 6. The average eigenstrain calculated form each results can be used for predicted curvature of specimen. By linking resolution of each eigenstrain, the increasing trend can be created as a function that used for prediction in different thickness of specimens.

The specimen of alumina ceramic $\left(\mathrm{Al}_{2} \mathrm{O}_{3}\right)$ is designed as $30 \mathrm{~mm}$ (wide) x $30 \mathrm{~mm}$ (length). The total thickness of $\mathrm{Al}_{2} \mathrm{O}_{3}$ is $4 \mathrm{~mm}$ and $8 \mathrm{~mm}$ respectively. The number of film layers is 200 layers and 400 layers. Each layer of the thickness was set up to $20 \mu \mathrm{m}$ for $3 \mathrm{~S}$. The measurement of alumina ceramic can be separated in two parts from sintering. For sintering process, furnace temperature was raised to $1600{ }^{\circ} \mathrm{C}$ at a rate of $5{ }^{\circ} \mathrm{C} / \mathrm{min}$ and was retained for 2 hours. Before sintering the product, the Young's modulus was around form 13.2 GPa to14.5 GPa, which are depended on the thickness of specimen. The average of Young's modulus is $238.27 \mathrm{GPa}$ after sintering process. The total shrinkage of $\mathrm{Al}_{2} \mathrm{O}_{3}$ is $79 \%$ after post-process. The curvature for each specimen is $292.3 \mathrm{~mm}$ and $253.85 \mathrm{~mm}$. The changed of curvature is not larger than that in photopolymer resin. By calculated from eigenstrain-thickness function, the average of eigenstrain can be obtained which is $3.91 \times 10^{-4}$ and $4.51 \times 10^{-4}$ respectively. The prediction of curvature using average eigenstrain is closed to the measurement. However, the result of eigenstrain was contained extrinsic and intrinsic phenomena that are shrinkage and phase transformation. This is not easily to detect those eigenstrain individually. Still, the eigenstrain can be simply described the bending system in two effects.

\section{Summary}

It is hardly to obtain eigenstrain and curvature at the same time. Therefore, in this paper, a semiempirical procedure for 3D printing was introduced. This method relies on an experimental procedure to determine the eigenstrain. The eigenstrain-thickness is available to understand the bending system for $3 \mathrm{D}$ printing product. This result can be useful for predict curvature and know the thickness would be affected to the product bending. In this study, different of material has different Young's modulus that need to establish a reasonable experimental process. Shrinkage can be roughly described one of the reason that caused the product bending. More detail reasons can be found from preprocessing which is included the mixture of material, the slurry mixing, partial size of the material and the power of UV light. For the material of alumina ceramic, sintering process is an important process to discuss. Phase transformation is one of resolution that caused bending system. In present study, the eigenstrain of $\mathrm{Al}_{2} \mathrm{O}_{3}$ is described for total eigenstrain that thermal stress was included in the product. As the reason discussed above, more detail should be investigated in the future.

\section{Acknowledgments}

This research was sponsored by Ministry of Science and Technology R.O.C under contract MOST103-2622-E-027-029-CC2.

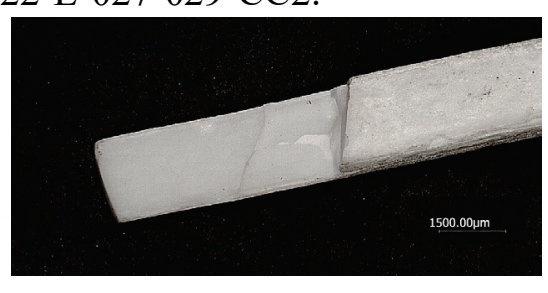

(a)

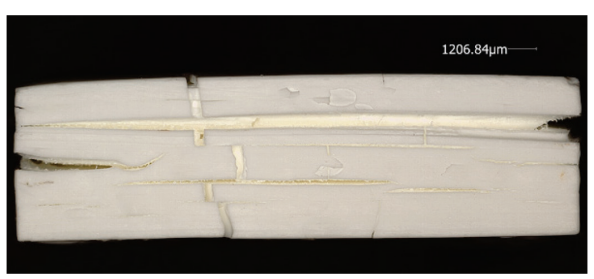

(b)

Fig. 1 (a) The inner force caused the quality of product unstable and cracked form internal; (b) The inferior attachment between each layer of product appeared along the boundary of alumina ceramic after sintering. 
(a)

(b)
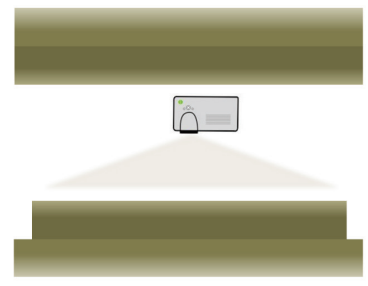

(c)

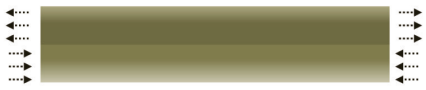

(d)

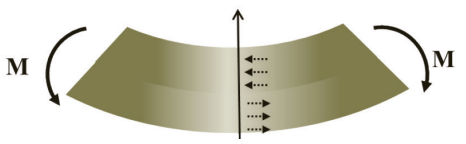

Fig. 2 After finish the printing, bending stress develop to balance bending moment. (a) Initial stress free condition (costing slurry); (b) constrained strain (mask exposing); (c) constrained inplane strain; (d) banding induced by asymmetric stresses (complete rigid part)

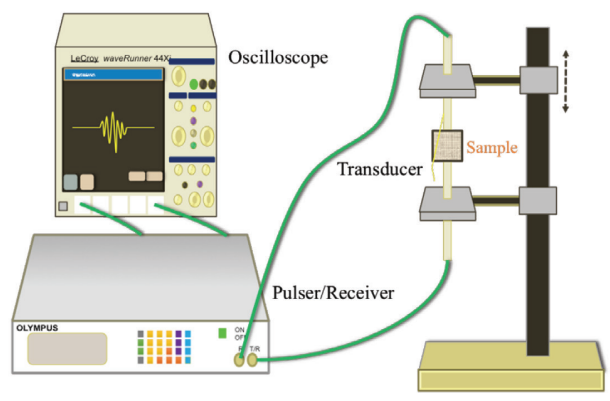

Fig. 3 The illustration for ultrasound signal generator.

Table 1. The initial experimental data of photopolymer resin.

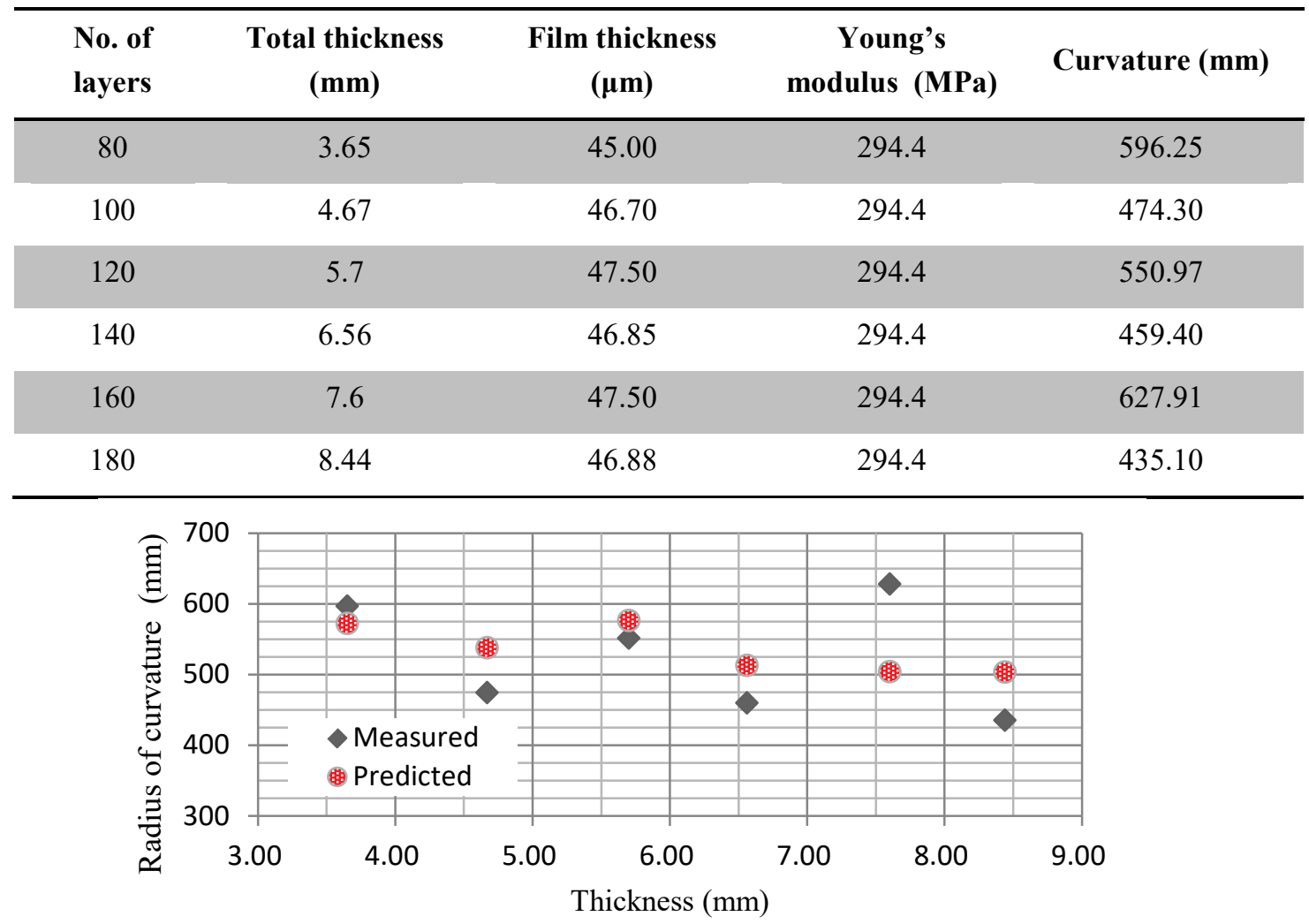

Fig. 5 Comparison of measured and predicted radius of curvature in 6 different thickness. 


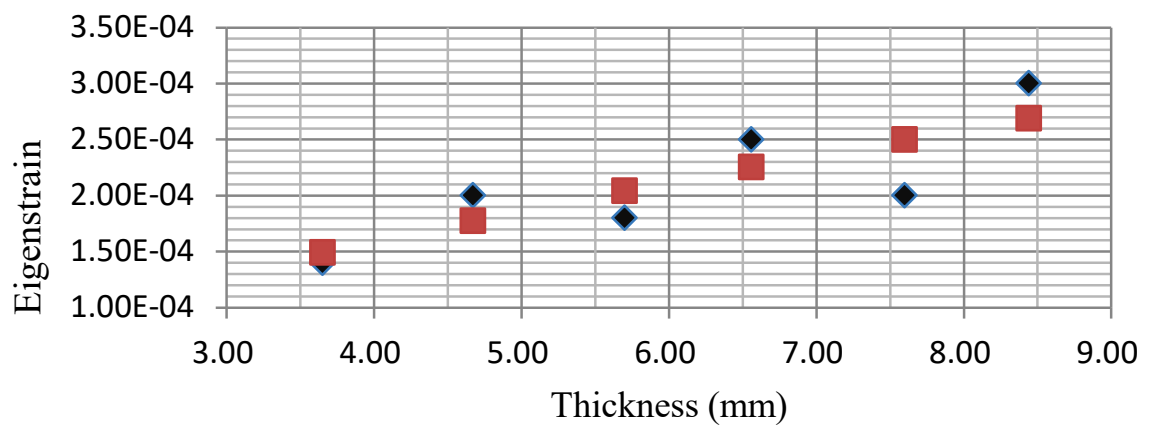

Fig. 6 Comparison of measured and predicted eigenstrain in 6 different thickness.

\section{References}

[1] Mustafa Megahed, Hans-Wilfried Mindt, Narcisse N'Dri, Hongzhi Duan, Olivier Desmaison, Metal additive-manufacturing process and residual stress modeling, Int. Materials and Manufacturing Innovation, (2016).

[2] R.H. Saul, Effect of GaAsx P1-x transition zone-GaAs substrates, J. Appl. 40, pp. 3272 (1969). https://doi.org/10.1063/1.1658174

[3] G.H. Olsen, M. Ettenberg, Calculate stresses in multilayered heteroepitaxial structures, J. Appl. 48, pp. 2543 (1977). https://doi.org/10.1063/1.323970

[4] C.H. Hsueh, Modeling of elastic deformation of multilayers due to residual stresses and external bending. J. Appl., pp. 9652 (2002).

[5] Jia-Chang Wang, A novel fabrication method of high strength alumina ceramic parts based on solvent-based slurry stereolithography and sintering, Int. J. Precis. Eng. Man., 14, (2013) 485 - 491. https://doi.org/10.1007/s12541-013-0065-3

[6] S. Timoshenko, Analysisof bi-metal thermostats, J. Opt. Soc. Amer., vol. 11, pp. 233-255 (1925). https://doi.org/10.1364/JOSA.11.000233

[7] A. Ni, D. Sherman, R. Ballarini, H. Kahn, B. Mi, S. M. Phillips, A. H. Heuer, Optimal design of multilayered polysilicon films for prescribed curvature, J. Materials Sci., vol. 38, pp. 4169 (2003). https://doi.org/10.1023/A:1026333707011

[8] Viktorov I.A., Rayleigh and lamb waves: physical theory and applications, Plenum Press, New York (1967). https://doi.org/10.1007/978-1-4899-5681-1 